\title{
Fatores de risco de óbito neonatal em região do interior paulista, Brasil
}

\author{
Risk factors for neonatal death in an inland region \\ in the State of São Paulo Brazil
}

Fernanda Cristina Manzini Sleutjes ${ }^{1}$

Cristina Maria Garcia de Lima Parada ${ }^{2}$

Maria Antonieta de Barros Leite Carvalhaes ${ }^{2}$

Milena Jamas Temer ${ }^{2}$

${ }^{1}$ Faculdade Sudoeste Paulista de Avaré. Av. Prof. Celso Ferreira da Silva 1001, Jardim Europa I. 18707-150 Avaré SP Brasil. fernandinhamanzini@ gmail.com

${ }^{2}$ Departamento de Enfermagem, Faculdade de Medicina de Botucatu, Universidade Estadual Paulista. São Paulo SP Brasil.

\begin{abstract}
The main objective of this study was to identify risk factors for neonatal death in an inland region of the State of São Paulo. A casecontrol study was conducted using a case group of 162 child deaths that occurred in 2009 in the state's VI Regional Health Department - Bauru. The control group consisted of 324 children selected from the Live Births Information System database who shared the same birth date and city of residence. Univariate and hierarchical multiple logistic regression analyses were performed to identify the factors associated with neonatal death by calculating crude odds ratios adjusted for potential confounders and respective 95\% confidence intervals. Results: The likelihood of neonatal death was greater among women who had had a history of infant death $(O R=24.97$, $C I=12.20$ to 51.10) and who had had only up to three antenatal appointments $(O R=11.40, C I$ $=5,92$ to 21.93), and among infants born at less than 28 weeks of gestation $(O R=168.00, C I=$ 49.63 to 568.66). The influence of birth weight was also observed among newborns weighing under 1,500g. Conclusions: This study identified five independent risk factors for neonatal death, the most notable of which is maternal history of neonatal death, which has not been properly acknowledged as a risk factor by previous studies. Key words Infant mortality, Epidemiology, Antenatal care, Childbirth
\end{abstract}

Resumo $O$ objetivo deste artigo é identificar os fatores de risco de óbito neonatal em região do interior paulista. Estudo de casos e controles. $O$ grupo casos foi constituído por 162 crianças/óbitos neonatais ocorridos em 2009 na região do Departamento Regional de Saúde VI- Bauru/SP. Compuseram o grupo controle 324 crianças selecionadas do Sistema de Informações de Nascidos Vivos dentre aquelas com o mesmo ano de nascimento $e$ município de residência. Para identificar os fatores associados ao óbito neonatal, realizou-se análise de regressão logística univariada e múltipla hierarquizada, estimando-se as razões de odds (e respectivos intervalos de confiança de 95\%) brutas e ajustadas para potenciais fatores de confusão. Apresentaram maior chance de óbito os neonatos cujas mães tinham história de óbito infantil, realizaram até três consultas pré-natais e idade gestacional inferior a 28 semanas. A influência do peso ao nascer foi observada apenas entre crianças com peso inferior a $1500 \mathrm{~g}$. Este estudo identificou, de maneira independente, cinco fatores de risco para o óbito neonatal, com destaque para a história materna de óbito infantil anterior, fator ainda não valorizado em estudos prévios.

Palavras-chave Mortalidade infantil, Epidemiologia, Cuidado pré-natal, Parto 


\section{Introdução}

Dados da Organização Mundial de Saúde (OMS) revelam queda no número de mortes na infância em âmbito mundial, passando de 12,7 milhões de casos em 1990 para estimados 5,9 milhões em 2015. Apesar dos avanços, ainda é grande o número de mortes evitáveis, especialmente no período neonatal, responsável por aproximadamente $45 \%$ dos óbitos deste período, com estimativa de um milhão de mortes no dia do nascimento e perto de dois milhões na primeira semana de vida. Entre as principais causas, estão a prematuridade, a pneumonia e as complicações decorrentes do trabalho de parto, entre outras ${ }^{1}$.

No Brasil, nas últimas décadas, evidencia-se redução da mortalidade na infância bem superior à média global: em 1990, ocorriam 51 mortes por mil nascidos vivos, número que foi reduzido a 15 por mil nascidos vivos em 2015. Como ocorre globalmente, o período neonatal é o que representa a maior parcela desses óbitos no país, com taxa de nove por mil nascidos vivos no último ano citado ${ }^{1}$.

A mortalidade infantil e seus componentes neonatal e pós-neonatal associam-se a inúmeros fatores: sociais, comportamentais, biológicos, entre outros, que atuam de modo hierarquizado. Estudo teórico sobre fatores de risco de óbito neonatal apontou que modelos teórico-conceituais são relevantes porque podem integrar diferentes fatores e, ainda, elucidar as relações que se estabelecem entre eles na determinação de desfechos de saúde. As autoras apresentaram modelo em quatro níveis: um distal, dois intermediários e um proximal ${ }^{2}$.

Os fatores mais distais associados à mortalidade infantil são sociais, como a escolaridade e a idade materna, e estes atuam pela capacidade de influenciar outros, envolvidos na longa cadeia causal do óbito neonatal. A multiparidade, a ordem e o intervalo de nascimento e o número de consultas pré-natais inferior a seis estão entre os determinantes intermediários e o baixo peso ao nascer e o escore de Apgar inferior a sete são considerados fatores de risco mais proximais ${ }^{3}$.

Conhecer e compreender os processos que resultam em óbito infantil é fundamental para a oferta de assistência de qualidade e efetiva ao binômio mãe-filho. Considerando-se as variações inter e intrarregionais observadas ${ }^{4}$, identificar seus determinantes em contextos específicos é medida importante para a definição de políticas e intervenções preventivas ou relacionadas ao cuidado à saúde infantil, justificando análises voltadas ao contexto regional. Assim, o presente estudo teve por objetivo identificar os fatores de risco de óbito neonatal em região do interior paulista. Em especial, buscou-se identificar os intermediários, relacionados às gestações pregressas, condições obstétricas e atenção pré-natal, menos discutidos no contexto nacional, embora, em geral, sejam passíveis de intervenções por parte dos serviços de saúde.

\section{Métodos}

Trata-se de estudo do tipo caso-controle, realizado no âmbito dos 68 municípios que compõem o Departamento Regional de Saúde (DRS) VI Bauru.

O DRS VI possui dois Grupos de Vigilância Epidemiológica (GVE) e, considerando-se o cuidado no ciclo gravídico puerperal, todos os municípios deste Departamento oferecem atendimento às gestantes de baixo risco. $\mathrm{O}$ atendimento ao parto e o cuidado pré-natal e neonatal especializados, porém, estão disponíveis apenas nos municípios-sede de um de seus cinco Colegiados de Gestão Regional: Avaré, Bauru, Botucatu, Jaú ou Lins.

O grupo caso foi constituído por 162 óbitos neonatais investigados, $84,8 \%$ do total de óbitos (191 casos) ocorridos no ano de 2009 no DRS VI. O grupo controle foi constituído por 324 neonatos nascidos no mesmo ano e em municípios de ocorrência dos casos, selecionando-se as duas crianças seguintes ao caso no registro do Sistema de Informações de Nascidos Vivos (SINASC). A estratégia de inclusão de dois casos para cada controle visou aumentar o poder estatístico do estudo, considerando que o número disponível de casos já estava previamente definido. Com esse tamanho de amostra, o estudo teve poder estatístico de $80 \%$ para detectar razão de chance igual ou superior a 2,5, com nível de significância de 5\% para fatores de exposição com frequências variando entre 20 e $80 \%$.

Considerando a fonte das informações utilizadas, cabe indagar sobre sua qualidade. Com o intuito de gerar dados confiáveis, os sistemas nacionais de informação estão em constante aperfeiçoamento, tanto em relação à cobertura quanto à qualidade dos dados ${ }^{5,6}$. Tem sido apontada a crescente qualidade dos dados do SINASC no país e estudo publicado em 2015 encontrou, para a região sudeste, percentual de completude dos dados acima de $99 \%{ }^{6}$. Não existem dados disponíveis para a DRS VI e não foi possível viabilizar 
a avaliação de todas as variáveis utilizadas no presente estudo, mas fala a favor da qualidade destas o fato de que, para a grande maioria, foram baixas as frequências de informações ignoradas, especialmente por que na região quase a totalidade dos nascimentos ocorrem em ambiente hospitalar, situação favorável à alimentação do Sistema de Informações.

Ainda sobre qualidade dos dados, recente estudo avaliou o registro de óbitos perinatais no SIM em um dos municípios sede de Polo de Gestão Regional da DRS VI e identificou taxa de concordância de $80 \%$ entre os dados constantes no sistema e os obtidos de forma independente, para a pesquisa. Embora voltado à avaliação do SIM, esses resultados também falam a favor da qualidade dos dados do SINASC utilizados neste estudo, devido à existência, na região, de equipes unificadas para alimentação de ambos os sistemas $^{7}$.

Destaca-se que antes de 2008 não havia instrumento padronizado para a investigação dos óbitos neonatais ocorridos no DRS VI, tampouco articulação entre os serviços de assistência materno-infantis municipais e regionais, dificultando o trabalho conjunto dos municípios de residência da criança e de ocorrência do óbito, resultando em cobertura de investigação de apenas 53\% dos casos e ainda de forma parcial. Em 2008, a partir de ações de educação permanente, buscou-se qualificar profissionais dos serviços de vigilância epidemiológica para a investigação do óbito, padronizar a ficha de investigação e construir fluxo de investigação que compreendesse toda a rede de saúde materno infantil. Assim, o presente estudo foi realizado após as citadas intervenções, que resultaram em qualificação das investigações e ampliação de sua cobertura.

A coleta de dados foi realizada em 2010, por uma das pesquisadoras, a partir das fichas de investigação de óbitos infantis do ano de 2009 fornecidas pelos GVE e do banco do SINASC. Os dados foram digitados no software Access e analisados no software estatístico SPSS versão 15.0.1

Foram investigadas associações entre óbito neonatal (variável dependente) e as seguintes variáveis explanatórias: anos de aprovação escolar da mãe (oito ou mais, até sete), idade materna em anos (20 a 34, até 19, 35 ou mais), presença de companheiro ( $\operatorname{sim}$, não), óbito infantil anterior (não, sim), filhos vivos (um a três, nenhum, quatro ou mais), tipo de gravidez (simples, dupla ou mais), número de consultas pré-natais (sete ou mais, quatro a seis, zero a três), tipo de parto (vaginal, cesárea), idade gestacional no parto em semanas ( 37 ou mais, 28 a $36,<28$ ), peso ao nascer em gramas (2.500 ou mais, 1.500 a 2.499 , $<1.500$ ) e índice de Apgar no primeiro minuto de vida (sete ou mais, quatro a seis, zero a três).

Realizou-se análise de regressão logística univariada e múltipla hierarquizada, estimando-se as razões de odds (e respectivos intervalos de confiança de 95\%) brutas e ajustadas para potenciais fatores de confusão. A análise múltipla foi guiada por modelo teórico de determinação (Figura 1), construído para a presente pesquisa e tendo como base o proposto por Lima et al. ${ }^{2}$ e o utilizado em estudo desenvolvido no nordeste do Brasil $^{8}$, sendo constituído por quatro blocos de variáveis: o bloco um (fatores mais distais) contém as variáveis sociodemográficas (escolaridade e idade materna); do bloco dois constam variáveis relativas às gestações pregressas (óbito infantil anterior e número de filhos vivos); o bloco

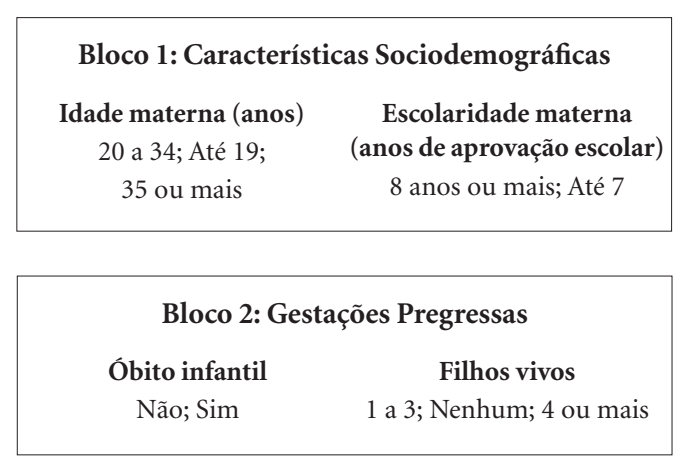

\begin{tabular}{|cc|}
\hline Bloco 3: Condição Obstétrica e Atenção Pré-Natal \\
Tipo de gravidez & Consultas pré-natais \\
Simples; Dupla & 7 ou mais; 4 a $6 ; 0$ a 3 \\
& \\
Tipo de parto & Idade gestacional no \\
Vaginal; Cesárea & parto (semanas) \\
& 37 ou mais; 28 a 36; $<28$ \\
\hline
\end{tabular}

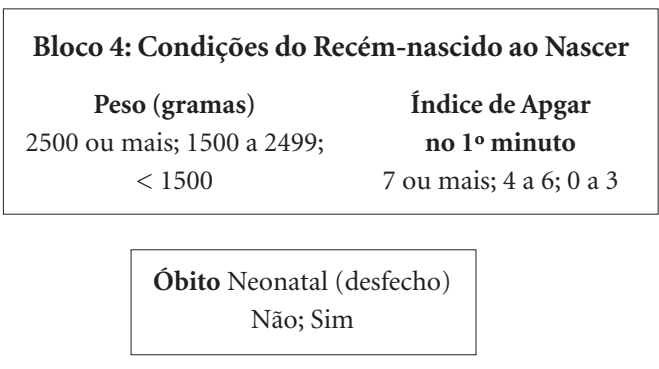

Figura 1. Modelo teórico para análise dos determinantes do óbito neonatal. 
III contém variáveis relativas às condições obstétricas e à atenção pré-natal (tipo de gravidez, número de consultas pré-natais, tipo de parto e idade gestacional no momento do parto) e no bloco quatro estavam as variáveis do recém-nascido (peso ao nascer e índice de Apgar no primeiro minuto de vida).

Para entrar na análise multivariada como potencial fator de confusão das variáveis do mesmo bloco ou dos inferiores, adotou-se $\mathrm{p}<$ 0,20 , exceto no caso da escolaridade, mantida no modelo independente do valor, para expressar nível socioeconômico, reconhecido como o determinante mais distal do desfecho em estudo. Para ser identificado como fator de risco de óbito neonatal, considerou-se como valor crítico $\mathrm{p}<$ 0,05 , avaliado pelo teste Wald, após ajuste para os fatores do mesmo bloco e precedentes.

O Projeto desta investigação foi aprovado pelo Comitê de Ética em Pesquisa da Faculdade de Medicina de Botucatu e respeitou todos os preceitos de estudos realizados com seres humanos.

\section{Resultados}

A Tabela 1 apresenta as características de casos e controles e os resultados das análises univariadas. Apresentaram maior chance de óbito os neonatos cujas mães tinham história de óbito infantil (OR $=24,97$, IC $=12,20-51,10)$, realizaram até três consultas pré-natais $(\mathrm{OR}=11,40$, IC $=5,92-21,93)$ ou entre quatro e seis consultas $(\mathrm{OR}=4,77, \mathrm{IC}=2,90-7,58)$ e tinham idade gestacional inferior a 28 semanas $(\mathrm{OR}=168,00$, IC $=49,63-568,66)$ ou entre 28 e 36 semanas $(\mathrm{OR}=$ $9,85, \mathrm{IC}=5,63-17,24)$. Com relação às condições do recém-nascido no parto, houve associação do óbito com o peso ao nascer: entre 1.500 e 2.499 gramas (OR $=5,34$, IC $=2,99-9,54)$ e abaixo de 1.500 gramas $(\mathrm{OR}=103,35, \mathrm{IC}=42,33-252,34)$ e com o índice de Apgar no primeiro minuto de vida: se entre zero e três $(\mathrm{OR}=47,18, \mathrm{IC}=23,00$ $96,77)$ ou entre quatro e seis $(\mathrm{OR}=27,07, \mathrm{IC}=$ 12,78-57,32).

Independentemente da escolaridade e do número de filhos vivos, a história materna de óbito infantil anterior aumentou o risco de óbito neonatal em 29 vezes ( $\mathrm{IC}=13,77-61,67)$. Também, ajustado pelos blocos precedentes e pelo tipo de parto, menos de seis consultas pré-natais associou-se de forma independente a óbito neonatal, aumentando sua chance entre 11 e 15 vezes. $\mathrm{O}$ risco de óbito associado a nascer extremamente prematuro (abaixo de 28 semanas de gestação) foi altíssimo: OR = 261; IC 95\% = 52,26-1310,10; nascer entre 28 e 36 semanas de idade gestacional multiplicou por seis a chance de óbito. Da mesma forma, exerceram efeito independente dos fatores precedentes sobre a chance de óbito neonatal as variáveis mais proximais: muito baixo peso ao nascer (menos de 1.500 gramas) e índice de Apgar inferior a sete no primeiro minuto de vida (Tabela 2).

\section{Discussão}

O presente estudo permitiu conhecer fatores associados ao óbito neonatal em ampla região do Estado de São Paulo. Foram identificados cinco fatores associados ao maior risco de óbito neonatal, dos quais três são do bloco de fatores intermediários, de acordo com o modelo teórico utilizado: história de óbito infantil, número de consultas pré-natais e prematuridade. O primeiro, embora não possa ser modificado, pode ter seu efeito atenuado pela qualidade do cuidado no período pré-natal e deveria ser utilizado como marcador de risco para cuidado pré-natal especializado; os demais são passíveis de intervenções durante a gestação e também estão ligados à qualidade do cuidado neste período. Fatores de risco mais proximais foram o baixo peso ao nascer, intimamente associado à prematuridade e o baixo índice de Apgar no primeiro minuto de vida, que também está associado à qualidade do cuidado, agora durante o trabalho de parto e o parto. Esse conhecimento indica que aumentar a qualidade da atenção à saúde (no pré-natal e no parto) deve ser prioridade na alocação de recursos para a saúde na região, com vistas à prevenção do óbito neonatal.

O fator de risco com maior efeito sobre a chance de óbito neonatal foi prematuridade, confirmando extensa literatura. Este é um problema global: recente revisão sistemática da literatura apontou o nascimento pré-termo como principal causa de morte não apenas infantil, mas na infância, à frente, inclusive, da pneumonia, que até então ocupava esta posição'. Assim como na presente investigação, estudo prospectivo de coorte, de caráter nacional, realizado na Holanda, indicou mortalidade neonatal fortemente associada à idade gestacional, variando de 546 por mil entre os nascidos com 25 semanas e 18 por mil quando se considerou nascidos com 31 semanas de idade gestacional ${ }^{10}$.

Se não é novidade que prematuridade é a principal causa de óbito neonatal, cabe aqui des- 
Tabela 1. Associação bruta entre óbitos neonatais e variáveis socioeconômicas, relativas à gestação pregressa, condições obstétricas e de nascimento da criança. DRS VI-Bauru/SP, Brasil, 2009.

\begin{tabular}{|c|c|c|c|c|}
\hline Variáveis & $\begin{array}{c}\text { Controles } \\
\mathrm{N}^{\mathrm{o}}(\%)\end{array}$ & $\begin{array}{c}\text { Casos } \\
\mathrm{N}^{\circ}(\%) \\
\end{array}$ & $\mathbf{p}$ & OR(IC95\%) \\
\hline \multicolumn{5}{|l|}{ Bloco I: Sociodemográfico } \\
\hline \multicolumn{5}{|l|}{ Escolar idade materna } \\
\hline 8 anos ou mais & $243(75,0)$ & $102(77,9)$ & & 1,00 \\
\hline Até 7 anos & $81(25,0)$ & $29(22,1)$ & 0,520 & $0,85(0,52-1,38)$ \\
\hline \multicolumn{5}{|l|}{ Idade materna (anos) } \\
\hline 20 a 34 & $213(65,8)$ & $89(65,9)$ & & 1,00 \\
\hline Até 19 & $72(22,2)$ & $31(23,0)$ & 0,904 & $1,03(0,63-1,67)$ \\
\hline 35 ou mais & $39(12,0)$ & $15(11,1)$ & 0,801 & $0,92(0,48-1,75)$ \\
\hline \multicolumn{5}{|l|}{ Bloco II: Gestações Pregressas } \\
\hline \multicolumn{5}{|l|}{ História de óbito infantil } \\
\hline Não & $290(96,7)$ & $72(53,7)$ & & 1,00 \\
\hline Sim & $10(3,3)$ & $62(46,3)$ & $<0,001$ & $24,97(12,20-51,10)$ \\
\hline \multicolumn{5}{|l|}{ Filhos vivos } \\
\hline 1 a 3 & $177(56,5)$ & $67(50,0)$ & & 1,00 \\
\hline Nenhum & $123(39,3)$ & $63(47,0)$ & 0,152 & $1,35(0,85-2,04)$ \\
\hline 4 ou mais & $13(4,2)$ & $4(3,0)$ & 0,725 & $0,81(0,26-2,58)$ \\
\hline \multicolumn{5}{|c|}{ Bloco III: Condições Obstétricas } \\
\hline \multicolumn{5}{|c|}{ Tipo de gravidez } \\
\hline Simples & $314(96,9)$ & $126(96,9)$ & & 1,00 \\
\hline Dupla & $10(3,1)$ & $4(3,1)$ & 0,996 & $0,99(0,30-3,23)$ \\
\hline \multicolumn{5}{|l|}{ Consultas pré-natais } \\
\hline 7 ou mais & $228(70,6)$ & $35(28,0)$ & & 1,00 \\
\hline 4 a 6 & $75(23,2)$ & $55(44,0)$ & $<0,001$ & $4,77(2,90-7,85)$ \\
\hline 0 a 3 & $20(6,2)$ & $35(28,0)$ & $<0,001$ & $11,40(5,92-21,93)$ \\
\hline \multicolumn{5}{|l|}{ Tipo de parto } \\
\hline Vaginal & $147(45,4)$ & $72(52,9)$ & & 1,00 \\
\hline Cesárea & $177(54,6)$ & $64(47,1)$ & 0,138 & $0,73(0,49-1,10)$ \\
\hline \multicolumn{5}{|l|}{ Idade gestacional (semanas) } \\
\hline 37 ou mais & $279(86,1)$ & $31(23,3)$ & & 1,00 \\
\hline 28 a 36 & $42(13,0)$ & $46(34,6)$ & $<0,001$ & $9,85(5,63-17,24)$ \\
\hline$<28$ & $3(0,9)$ & $56(42,1)$ & $<0,001$ & $168,00(49,63-568,66)$ \\
\hline \multicolumn{5}{|c|}{ Bloco IV: Condições do Neonato } \\
\hline \multicolumn{5}{|l|}{ Peso (gramas) } \\
\hline 2500 ou mais & $278(85,8)$ & $39(25,0)$ & & 1,00 \\
\hline 1500 a 2499 & $40(12,3)$ & $30(19,2)$ & $<0,001$ & $5,34(2,99-9,54)$ \\
\hline$<1500$ & $6(1,9)$ & $87(55,8)$ & $<0,001$ & $103,35(42,33-252,34)$ \\
\hline \multicolumn{5}{|l|}{ Apgar 1 minuto } \\
\hline 7 ou mais & $297(92,6)$ & $32(25,0)$ & & 1,00 \\
\hline 4 a 6 & $12(3,7)$ & $35(27,3)$ & $<0,001$ & $27,07(12,78-57,32)$ \\
\hline 0 a 3 & $12(3,7)$ & $61(47,7)$ & $<0,001$ & $47,18(23,00-96,77)$ \\
\hline
\end{tabular}

tacar que a situação brasileira não é confortável: o Brasil está entre as 10 nações com maior proporção de nascimentos pré-termos ${ }^{11}$, sendo a maior parte destes considerados reduzíveis por adequada atenção à mulher na gestação ${ }^{12}$. A prevenção de partos prematuros depende de investimentos 
Tabela 2. Associação entre óbito neonatal e variáveis relacionadas às gestações pregressas (Bloco II), condições obstétricas (Bloco III) e condições do neonato (bloco IV) considerando-se valores de odds ratio ajustados. DRS VI-Bauru/ SP, Brasil, 2009.

\begin{tabular}{|c|c|c|}
\hline Variáveis & $\mathrm{p}$ & OR(IC95\%) \\
\hline \multicolumn{3}{|l|}{ Bloco II: Gestações } \\
\hline \multicolumn{3}{|l|}{ Pregressas* } \\
\hline \multicolumn{3}{|l|}{$\begin{array}{l}\text { História de óbito } \\
\text { infantil }\end{array}$} \\
\hline Não & & 1,00 \\
\hline Sim & $<0,001$ & $29,14(13,77-61,67)$ \\
\hline \multicolumn{3}{|l|}{ Filhos vivos } \\
\hline 1 a 3 & & 1,00 \\
\hline Nenhum & 0,092 & $1,54(0,93-2,57)$ \\
\hline 4 ou mais & 0,312 & $0,43(0,08-2,19)$ \\
\hline \multicolumn{3}{|l|}{ Bloco III: Condições } \\
\hline \multicolumn{3}{|l|}{ Obstétricas $^{\dagger}$} \\
\hline \multicolumn{3}{|c|}{ Consultas pré-natais } \\
\hline 7 ou mais & & 1,00 \\
\hline 4 a 6 & $<0,001$ & $11,40(3,95-32,87)$ \\
\hline 0 a 3 & $<0,001$ & $15,45(4,29-55,65)$ \\
\hline \multicolumn{3}{|l|}{ Tipo de parto } \\
\hline Vaginal & & 1,00 \\
\hline Cesárea & 0,686 & $1,88(0,51-2,74)$ \\
\hline \multicolumn{3}{|l|}{$\begin{array}{l}\text { Idade gestacional } \\
\text { (semanas) }\end{array}$} \\
\hline 37 ou mais & & 1,00 \\
\hline 28 a 36 & $<0,001$ & $6,78(2,74-16,78)$ \\
\hline$<28$ & $<0,001$ & $261,67(52,26-1310,10)$ \\
\hline \multicolumn{3}{|c|}{$\begin{array}{l}\text { Bloco IV: Condições do } \\
\text { Neonato }\end{array}$} \\
\hline \multicolumn{3}{|c|}{ Peso (gramas) } \\
\hline 2500 ou mais & & 1,00 \\
\hline 1500 a 2499 & 0,980 & $0,98(0,20-4,77)$ \\
\hline$<1500$ & 0,032 & $11,34(1,23-103,81)$ \\
\hline \multicolumn{3}{|l|}{ Apgar 1 minuto } \\
\hline 7 ou mais & & 1,00 \\
\hline 4 a 6 & $<0,001$ & $11,65(2,66-50,88)$ \\
\hline 0 a 3 & $<0,001$ & $46,06(11,30-187,77)$ \\
\hline
\end{tabular}

* Bloco II: ajustado pela escolaridade e variáveis do mesmo bloco. †Bloco III: ajustado pela escolaridade, variáveis do bloco II e pelas variáveis do mesmo bloco. ¥Variável não incluída nos ajustes do Bloco IV. plicações do parto pré-termo podem ser reduzidas em mais de três quartos com a oferta de ações básicas, como aquecimento, apoio ao aleitamento materno e cuidados básicos para infecções e dificuldades respiratórias, desde que os profissionais de saúde sejam qualificados para o atendimento a esses bebês e o serviço de saúde disponha dos equipamentos e materiais necessários.
Intimamente relacionado à idade gestacional ao nascimento, o peso ao nascer é em geral beneficiado com ações de prevenção do parto prematuro. Neste estudo, de maneira independente, apenas o peso inferior a 1.500 gramas foi relevante. A ausência de efeito negativo independente na faixa de peso entre 1.500 e 2.499 gramas é provavelmente decorrente do avanço tecnológico ocorrido nos últimos anos e que atualmente viabiliza a sobrevida desses bebês.

A principal contribuição deste estudo foi identificar a história materna de óbito infantil anterior como fator de risco independente de óbito neonatal. Embora esta condição não seja modificável, cabe, também nestes casos, intervenção no período pré-natal, pois mulheres nesta situação deveriam ser consideradas gestantes de risco. Porém, quando se consideram os fatores relacionados à história reprodutiva, adotados no Brasil para classificação de risco obstétrico, encontram-se apenas história materna de natimorto ou óbito perinatal ${ }^{13}$. Assim, sugere-se ampliar o conceito de perda anterior, incluindo história materna de filho falecido, independentemente da idade, como critério para recomendar o encaminhamento e ao menos uma avaliação da gestante em serviço especializado ou referência para gestação de risco, para que se decida o melhor local para seu seguimento pré-natal.

Ainda dentro dos fatores independentes passíveis de modificação por ações dos serviços de saúde, a baixa frequência de consultas de prénatal é condição modificável, especialmente pela captação precoce de todas gestantes de determinada área; garantia das consultas de retorno conforme prazos indicados ${ }^{13}$; busca ativa de faltosas; verificação se casos encaminhados estão sendo atendidos no serviço de referência e extinção da alta do pré-natal, que deve ser considerado efetivamente concluído apenas após a consulta de revisão de parto. Estas ações deverão ser iniciadas ou reforçadas nos serviços de atenção pré-natal da região estudada.

Porém, apesar da importância do seguimento pré-natal periódico, sabe-se que não basta avaliar quantitativamente o número de consultas de prénatal $^{13}$. Essa é uma limitação deste estudo, pois avaliou apenas o número e não a qualidade das consultas realizadas. Os autores desta investigação sugerem a realização de futuras pesquisas, voltadas a investigar o papel de indicadores da qualidade da atenção pré-natal como fatores de risco de morte neonatal, tais como: capacidade de predizer a ocorrência de desvios do crescimento intra-uterino; de evitar a sífilis congênita, 
pelo diagnóstico e tratamento precoces na gestação; de realizar a isoimunização Rh pela oferta de gamaglobulina à mãe no momento oportuno.

Neste estudo, independente de outros fatores, ter índice de Apgar inferior a sete no primeiro minuto de vida constituiu fator de risco para óbito no período neonatal. Apesar dos questionamentos quanto à sensibilidade, especificidade e capacidade deste índice predizer aspectos relativos à morbimortalidade, a curto e longo prazos ${ }^{14}$, é ainda muito utilizado em estudos que avaliam condições do recém-nascido nos primeiros minutos após o parto e na prática clínica, como indicador de algum grau de depressão neonatal ${ }^{15,16}$. O presente estudo confirma sua capacidade de indicar a vulnerabilidade do recém-nascido.

Assim como nesta, investigações nacionais e internacionais ${ }^{17,18}$ têm encontrado associação entre índice de Apgar baixo e mortalidade neonatal: em São José dos Campos/SP, estudo realizado com dados da Declaração de Nascidos Vivos e da Declaração de Óbito identificou risco de óbito neonatal três vezes maior entre recém-nascidos com índice de Apgar inferior a sete, quando comparados aos nascidos com índices maiores; na Escócia, estudo de base populacional realizado com mais de um milhão de nascimentos encontrou baixos valores de Apgar (0-3) no quinto minuto de vida fortemente associados ao risco de morte neonatal e infantil.

Para subsidiar ações visando à prevenção do óbito neonatal na região, sugere-se a realização de estudos que permitam identificar a qualidade da atenção ao parto e ao recém-nascido nela ofertada, por exemplo, avaliando se tecnologias demonstradamente úteis no parto foram incorporadas às maternidades existentes em sua área de abrangência; se a Rede de Atenção à Saúde tem viabilizado que o acompanhamento pré-natal e ao parto seja realizado nos serviços com a complexidade que cada caso exige, inclusive a partir de Central de Regulação; se as gestantes são acolhidas e atendidas, quando em trabalho de parto, na primeira maternidade que procuram; se as mulheres são efetivamente informadas sobre sua condição e todos os procedimentos a que serão submetidas, entre outros aspectos relevantes e que podem atestar a qualidade do cuidado.

Sendo este um estudo de casos e controles, análise temporal das associações tende a constituir limitação. Entretanto, neste caso, todas as variáveis associadas ao óbito corresponderam a eventos que antecederam a esse desfecho e as associações identificadas não estavam sujeitas ao viés de memória. Uma limitação que deve ser apontada, porém, é a utilização de fontes diferentes para a obtenção das informações de casos e controles, visto que dados dos casos foram obtidos das fichas de investigação de óbito neonatal e, no caso dos controles, foram utilizados dados secundários do SINASC. Quanto aos dados em si, como exposto em métodos, a coleta ocorreu logo após uma ação de educação permanente que visava justamente qualificar as equipes para a investigação do óbito infantil. Também, as equipes que registram dados no SINASC tinham treinamento recente e supervisão adequada, de modo que se pode supor sua boa qualidade, embora não tenha sido realizado na região estudo neste sentido.

\section{Conclusões}

Este estudo evidenciou cinco fatores de risco independentes para o óbito neonatal na região do DRS VI - Bauru/SP: prematuridade e baixo peso ao nascimento, especialmente em situações mais extremas, como abaixo de 28 semanas de gestação e entre neonatos com peso inferior a 1.500 gramas. O mesmo ocorreu com o índice de Apgar, já que a magnitude do efeito sobre o óbito neonatal foi quatro vezes maior para valores entre zero e três do que entre quatro e seis. Evidenciou, também, a importância de seguimento pré-natal com sete ou mais consultas e que a história materna de óbito infantil anterior deve ser valorizada, sugerindo-se que gestantes nesta situação sejam encaminhadas para avaliação em serviço especializado, pela forte associação com o óbito neonatal.

\section{Colaboradores}

FCM Sleutjes e CMGL Parada trabalharam a concepção e o delineamento ou a análise e interpretação dos dados e confecção final, MABL Carvalhaes e MJ Temer atuaram na redação do artigo ou na sua revisão crítica. 


\section{Referências}

1. World Health Organization (WHO). Estimates Developed by the UN Inter-agency Group for Child Mortality Estimation United. Geneva: WHO; 2015.

2. Lima S, Carvalho ML, Vasconcelos AGG. Proposta de modelo hierarquizado aplicado à investigação de fatores de risco de óbito infantil neonatal. Cad Saude Publica 2008; (8):1910-1916.

3. Geib LTC, Fréu CM, Brandão M, Nunes ML. Determinantes sociais e biológicos da mortalidade infantil em coorte de base populacional em Passo Fundo, Rio Grande do Sul. Cien Saude Colet 2010; 15(2):363-370.

4. Brasil. Ministério da Saúde (MS). Fundação Sistema Estadual de Análise de Dados. Resenha de Estatísticas Vitais do Estado de São Paulo [Internet]. São Paulo: SEADE; 2013 [acessado 30 Maio 2016]. Disponível em: http://www.seade.gov.br/produtos/midia/spdemografico/spdemog_ago2013.pdf

5. Brasil. Ministério da Saúde (MS), Parada CMGL, Sleutjes FCM, Escalante JJC, Carvalhaes MABL, Duarte MTC, Lima RB, Tonete VLP. Vigilância em Saúde materno-infantil: ação educativa para a redução de óbitos infantis com base na realidade epidemiológica de estados selecionados. Brasília: Editora do Ministério da Saúde; 2014.

6. Oliveira MM, Andrade SSCA, Dimech GS, Oliveira JCG, Malta DC, Rabelo Neto DL, Moura L. Avaliação do Sistema de Informações sobre Nascidos Vivos. Brasil, 2006 a 2010. Epidemiol. Serv. Saúde 2015; 24(4):629640.

7. Moraes, MF, Gonçalves IR, Tonon CR, Barbosa M, Duarte MTC, Parada CMGL. Surveillance system evaluation of the perinatal deaths in a hospital of reference. Proceedings of the 9th Encontro de Pós Graduação da FMB. 2016 May 18-20; Botucatu, São Paulo. Botucatu: Faculdade de Medicina de Botucatu; 2016.

8. Nascimento RM, Leite AJM, Almeida NMGS, Almeida PC, Silva CF. Determinantes da mortalidade neonatal: estudo caso-controle em Fortaleza, Ceará, Brasil. Cad Saude Publica 2012; 28(3):559-572.

9. Liu L, Oza S, Hogan D, Perin J, Rudan I, Lawn JE, Cousens S, Mathers C, Black RE. Global, regional and national causes of child mortality in 2000-13, with projections to inform post 2015 priorities: an update systematic analysis. Lancet 2015; 385(9966):430-440.

10. Ravelli ACJ, Schaaf JM, Mol BWJ, Tamminga P, Eskes M, Van der Post JAM, Abu-Hanna A. Antenatal prediction of neonatal mortality in very premature infants. Eur J Obstet Gynecol Reprod Biol 2014; 176:126-131.
11. World Health Organization (WHO). Born too soon: the global action report on preterm birth. Geneva: WHO; 2012.

12. Malta DC, Duarte EC, Almeida MF, Dias MAS. Lista de causas de mortes evitáveis por intervenções do Sistema Único de Saúde do Brasil. Epidemiol Serv Saude 2010; 19(2):173-176.

13. Brasil. Ministério da Saúde (MS). Atenção ao pré-natal de baixo risco. Brasília: Editora do Ministério da Saúde; 2013. (Cadernos de Atenção Básica, n. 32).

14. Bharti B, Bharti S. A review of the Apgar score indicated that contextualization was required within the contemporary perinatal and neonatal care framework in different settings. J Clin Epidemiol 2005; 58(2):121-129.

15. Kvestad E, Lie KK, Eskild A, Engdahl B. Sensorineural hearing loss in children: the association with Apgar score. A registry-based study of 392371 children in Norway. Int $J$ Pediatr Otorhrinolaryngol 2014; 78(11):1940-1944.

16. Terrell ML, Hartnett KP, Lim H, Wirth J, Marcus M. Maternal exposure to brominated flame retardants and infant Apgar scores. Chemosphere 2015; 118:178-186.

17. Lliodromiti S, Mackay DF, Smith GCS, Nelson SM. Apgar score and the risk of cause-specific infant mortality: a population-based cohort study. Lancet 2014; 384(9956):1749-55

18. Risso SP, Nascimento LFC. Fatores de risco para óbito neonatal obtidos pelo modelo de regressão multivariado de Cox. Rev Paul Pediatr. 2011; 29(2):208-13.

Artigo apresentado em 19/01/2016

Aprovado em 01/09/2016

Versão final apresentada em 03/09/2016 